
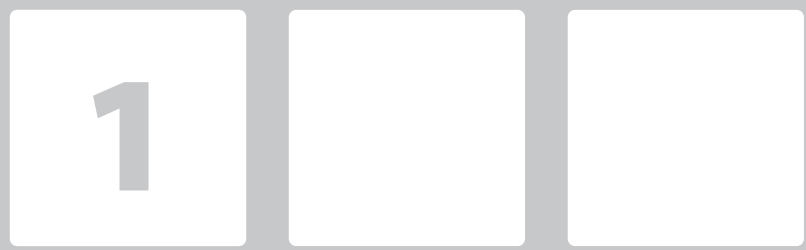

\title{
OS ANTECEDENTES DA COCRIAÇÃO DE VALOR EM PROGRAMAS DE INCENTIVO AO SEXO SEGURO: CONTRIBUIÇÕES DA LÓGICA DOMINADA POR SERVIÇO AO MARKETING SOCIAL
}

\section{The antecedents of value co-creation in safer sex incentive programs: contributions from Service-Dominated Logic to Social Marketing}

\section{Agnaldo Keiti Higuchi}

Universidade Federal dos Vales do Jequitinhonha e Mucuri - UFVJM/ FACSAE - Departamento de Administração, área finanças e marketing. email:agnaldo.higuchi@ufvjm.edu.br

\section{Ricardo Teixeira Veiga}

Universidade Federal de Minas Gerais/FACE - Centro de pesquisa e pós-graduação em Administração-CEPEAD, área marketing, estratégia e inovação.email:ricardo.necc@gmail.com

\section{Juliana Borges Martins}

Universidade Federal dos Vales do Jequitinhonha e Mucuri UFVJM/FACSAE - Departamento de Administração, área economia e marketing.email:juliana.borges@ufvjm.edu.br

\section{RESUMO}

Neste trabalho aborda-se a utilidade da Lógica Dominada por Serviço (LUSCH; VARGO, 2014), para o entendimento de fenômenos do Marketing Social, com ênfase na cocriação de valor pelo beneficiário de programas sociais voltados para a adoção do sexo seguro. Após pesquisa bibliográfica sobre o tema, elaborou-se modelo hierárquico contendo os constructos que envolvem a cocriação de valor e seus potenciais antecedentes. A partir do modelo foi criado o questionário, que foi aplicado a estudantes universitários na cidade de Teófilo Otoni-MG. Os resultados, obtidos por meio de Modelagem de Equações Estruturais, mostram que o constructo nível de atividades tem como antecedente o traço elementar abertura a experiências, enquanto que o constructo número de interações é explicado pelos traços elementares abertura a experiência, neuroticismo e necessidade de recursos materiais (negativamente).

Palavras-Chave: Lógica Dominada por Serviço. Marketing Social. Sexo seguro. Modelo 3M de Motivação e Personalidade.

\section{ABSTRACT}

In this work, the Service-Dominant Logic (LUSCH;VARGO, 2014) premises are applied in order to understand Social Marketing phenomena and processes, specifically on value creation by the beneficiary of safe sex programs. After bibliographical research a hierarchical model was elaborated containing the constructs involving value cocreation and its potential antecedents. From the model a questionnaire was created, which was applied to undergraduate students in the city of Teófilo Otoni-MG. The collected data were analyzed using Modeling Structural Equations. The results show that the construct level of activities has as antecedent the elemental trait openness to experiences, whereas the construct number of interactions is explained by the elemental traits opening to experience, neuroticism and need of material resources (negatively).

Key-words: Service - Dominant Logic. Social Marketing. Safe Sex. 3M Model of Motivation and Personality. 


\section{INTRODUÇÃO}

Segundo Hunt (2002), Marketing é a ciência comportamental das trocas, cujas bases teóricas incluem a economia, psicologia, sociologia, antropologia e teoria da decisão. As trocas podem ocorrer em diversos ambientes, e seu estudo aprofundado gera algumas ramificações: Marketing Digital, Marketing de Relacionamento, Marketing Esportivo, Marketing Social Corporativo, Marketing Social. No Marketing Social, as relações de troca não envolvem necessariamente valores monetários, pois o que se tenta "vender" ao beneficiário é uma ideia, uma proposta de mudança no comportamento (ANDREASEN, 1994).

À luz do exposto, considera-se a ideia da mudança de comportamento, ou a proposta de comportamento (PEATTIE; PEATTIE, 2003) como o produto trabalhado pelo Marketing Social, que, para não incorrer na chamada miopia em marketing, deve oferecer ao beneficiário a solução de um problema que esse quer resolver (LEVITT, 1985). De certa forma, o produto do Marketing Social "pode ser considerado convergente com o conceito mercadológico contemporâneo de produto, que destaca o resultado ou solução obtida" (VEIGA, 2014, p. 3).

A "venda" da proposta de mudança de comportamento no Marketing Social, entretanto, é apoiada por ferramentas empregadas no Marketing Comercial, adaptadas para o contexto social (ANDREASEN, 1994). Autores como Peattie e Peattie (2003) e Gordon (2012) argumentam que quando ferramentas do marketing comercial são empregadas em programas de marketing social corre-se o risco de construir um corpo teórico equivocado. Para Peattie e Peattie (2003), a aplicação de princípios e práticas do marketing comercial em programas sociais, considerando de forma superficial as diferenças entre o contexto comercial e social pode resultar em um corpo teórico de marketing social que não reflete a realidade onde é aplicado.

No caso específico de programas que envolvem questões de saúde, como a saúde sexual, há uma invasão, em certa medida, da privacidade do beneficiário. Este pode considerar essa situação constrangedora, por isso os aspectos emocionais, muitas vezes, são mais relevantes que os aspectos cognitivos no processo de tomada de decisão de consumo das ofertas dos serviços de saúde e dos programas sociais (CALERO-GIMENO; GALLARZA-GRANIZO, 2015).

Os autores acima sugerem a necessidade de alternativas teóricas mais alinhadas com o contexto encontrado nos fenômenos das mudanças voluntárias de comportamento social, que diferem do contexto das trocas comerciais. O contexto dos programas sociais é caracterizado pela ausência de uma troca direta entre fornecedor-consumidor, uma vez que a organização promotora do programa social não recebe contrapartida financeira do cliente.

Outra característica está no fato do produto trocado não beneficiar somente o cliente, mas a sociedade como um todo, pois ao fazer com que indivíduos abandonem comportamentos prejudiciais, os benefícios podem se estender para a comunidade. Por fim, no contexto dos programas sociais, a definição de papéis como produtor e consumidor fica dificultada, pois o consumidor pode ser tornar coprodutor ao encorajar outros indivíduos a mudar o comportamento (ANDREASEN, 2002).

Em suma, os autores parecem concordar que a lógica que enfatiza o produto não seria totalmente apropriada para descrever o fenômeno da mudança social buscada pelo marketing social, nem para analisar o contexto em que as mudanças ocorrem. Uma alternativa para auxiliar a elaboração de um corpo teórico dedicado ao marketing social é a Lógica Dominada por Serviço, proposta por Lusch e Vargo (2006), onde o foco está na troca de serviço entre atores, viabilizada pela integração de recursos de todos os atores envolvidos. Essa integração de recursos, influenciada por fatores individuais e sociais, possibilita a geração de benefícios e a cocriação de valor dentro do ecossistema de valor analisado.

Neste trabalho aborda-se a utilidade da Lógica Dominada por Serviço (VARGO; LUSCH, 2004), para o entendimento de fenômenos e processos do Marketing Social, com ênfase na cocriação de valor pelo consumidor-alvo dos programas em Marketing Social. A teoria presente na SDL pode fornecer subsídios para uma compreensão de fenômenos sociais e desenvolvimento de modelos gerenciais que auxiliem os gestores dos programas sociais (LUCA; HIBBERT; MCDONALD, 2015).

Tem-se como tema do trabalho, então, as possibilidades teóricas geradas pela aplicação da Lógica 
Dominada por Serviço - SDL no aprimoramento do corpo teórico do Marketing Social, usando como ponte entre a teoria mais geral e a aplicação empírica (BRODIE; SAREN; PELS, 2011) o Modelo Metateórico de Motivação e Personalidade - 3M de Mowen (2000). As propostas teóricas são analisadas empiricamente por meio de pesquisa entre estudantes que frequentam o curso de Administração da Universidade Federal dos Vales do Jequitinhonha e Mucuri no Campus do Mucuri-MG.

O problema abordado é a ausência de ferramentas gerenciais adequadas que auxiliem os gestores de programas em Marketing Social a aprimorar a efetividade dos programas. Para aumentar o arcabouço teórico atual sobre o tema, esse trabalho tem como objetivo propor um modelo de cocriação de valor, gerado a partir de pesquisa bibliográfica, e validar empiricamente o modelo aplicando o mesmo no estudo de um fenômeno do domínio do Marketing Social, no caso a adoção do uso frequente do preservativo. A questão da pesquisa seria: da perspectiva da SDL, o que se deve conhecer para maximizar o valor cocriado pelos beneficiários dos programas sociais?

Para responder a questão de pesquisa, esse estudo está estruturado da seguinte forma: inicialmente faz-se uma breve introdução do trabalho, indicando contexto e objetivos e justificativas. No tópico um expõe-se o Referencial Teórico, incluindo: (1) os fundamentos da Lógica Dominada por Serviço (Service Dominant logic - SDL); (2) limitações no atual corpo teórico do Marketing Social; (3) práticas de cocriação de valor e integração de recursos propostas pela SDL para um Marketing Social menos limitado; (4) as bases teóricas do Modelo Metateórico de Motivação e Personalidade de Mowen (2000).

No tópico dois apresenta-se o modelo conceitual-hipotético e as hipóteses da pesquisa. No tópico três descreve-se o método empregado na pesquisa, incluindo formas de aplicação e coleta de dados, forma de análise e critérios de comprovação de ajuste do modelo e de validade das análises. No tópico quatro é apresentada a análise dos resultados e, por fim, após a apresentação dos resultados são feitas as considerações finais.

\section{REFERENCIAL TEÓRICO}

\subsection{Introdução à Lógica Dominada por Serviço - SDL}

A SDL representa uma proposta de mudança de paradigma, da atual predominante Lógica dominada pelo Produto para uma Lógica dominada por Serviço, em que o termo Serviço passa a ser o denominador comum de trocas econômicas e não econômicas (VARGO; LUSCH, 2017).

Para organizar o corpo teórico e expressar os argumentos da SDL, Vargo e Lusch (2016) elaboraram as premissas fundamentais (ou fundacionais):

- (P1): serviço é a base fundamental da troca;

- (P2): troca indireta encobre a base fundamental de troca;

- (P3): bens são mecanismos de distribuição de serviços;

- (P4): recursos operantes são as fontes fundamentais de benefício estratégico;

- (P5): todas as economias são economias de serviço;

- (P6): valor é cocriado por múltiplos atores, sempre incluindo o beneficiário;

- (P7): atores não podem entregar valor, mas podem participar na criação e oferta de propostas de valor;

- (P8): uma visão centrada em serviço é inerentemente orientada para o beneficiário e relacional;

- (P9): todos os atores, sociais e econômicos são integradores de recursos;

- (P10): valor é unicamente e fenomenologicamente determinado pelo beneficiário;

- (P11): a cocriação de valor é coordenada por meio de instituições geradas pelos atores e por lógicas institucionais.

Estas premissas fundamentais fornecem um quadro para reexaminar e possivelmente aumentar o conhecimento sobre temas como inovação tecnológica e consolidação de práticas institucionalizadas (POHLMANN; KAARTEMO, 2017). Entretanto, nesse estudo o foco está no processo de troca e seu papel na sociedade (VARGO; LUSCH, 2016). Pode-se perceber que de acordo com as premissas 06, $07 \mathrm{e}$ 
10, apesar de diferentes atores proporem valor, este é determinado pelo beneficiário, o que traz implicações no modo como as empresas abordam o mercado e no modo como deveriam planejar seus programas e ações de Marketing. Os consumidores passam de recipientes passivos de mensagens e pesquisas a participantes ativos na criação de experiências (BARON et al., 2010).

$\mathrm{Na}$ SDL, Marketing pode ser visto como um potencializador de criação de valor pela firma e de sua entrega ao público, com ênfase na compreensão do potencial para cocriação presente nas experiências vividas nos encontros de serviço (PAYNE; STORBACKA; FROW, 2008; PRAHALAD; RAMASWAMY, 2004). Por isso, a prática do marketing precisa ir além da noção de valor transferido ao cliente durante a compra, para uma noção em que valor é criado conjuntamente com clientes (beneficiários) no contexto do uso.

O papel do marketing seria o de facilitar a integração de recursos e a cocriação de valor (BETTENCOURT; LUSCH; VARGO, 2014), fornecendo às empresas subsídios para que estas forneçam serviços e experiências que auxiliem os clientes a utilizar seus recursos (PAYNE; STORBACKA; FROW, 2008). Ou seja, deve-se superar a visão tradicional fornecedor-consumidor, para uma abordagem sistêmica de redes de atores interagindo em um ecossistema de serviço.

O ecossistema de serviço, na SDL, é definido como um sistema de relacionamentos de trocas diretas e indiretas entre os atores envolvidos. Amplia-se a díade produtor-consumidor, e adota-se a premissa de que nas trocas de serviço, atores integradores de recursos "se conectam por lógicas institucionais compartilhadas e mútua criação de valor" (LUSCH; VARGO, 2014, p. 167). Assim, no ecossistema de serviços todos os participantes direta e indiretamente envolvidos nas trocas passam a ser denominados atores integradores de recursos.

A integração de recursos se refere a como organizações, famílias e indivíduos integram e transformam competências micro especializadas em serviços complexos que são demandados no mercado, o qual realiza funções particulares do sistema de serviço para um beneficiário ou ator específico no sistema (PETERS et al., 2014). Percebe-se que a integração de recursos não é possível se estes se limitarem a materiais físicos, como matérias primas. Na SDL, recursos são as entidades tangíveis e intangíveis disponíveis utilizados pela firma para criar valor, sendo o serviço resultado da aplicação de recursos operantes (conhecimentos e habilidades) sobre recursos operáveis (máquinas e matérias-primas).

Os argumentos da SDL, entretanto, não possuem aceitação total por parte dos estudiosos em Marketing. Ela é criticada por apresentar conceito simplista e errôneo de valor, além de apresentar falta de argumentos para analisar fenômenos relacionados à cultura do consumo (HIETANEN; ANDÉHN; BRADSHAW, 2018). Apesar dessas críticas, esse trabalho adota o arcabouço teórico da SDL como base teórica do estudo.

\subsection{Limitações no atual corpo teórico do Marketing Social}

Entre as diferentes definições encontradas na literatura está a de Andreasen (1994, p.110), que conceitua o Marketing Social como

a adaptação de tecnologias do marketing comercial aplicadas em programas planejados para influenciar o comportamento voluntário de um público-alvo para gerar melhorias no bem estar pessoal, assim como no da sociedade da qual este público faz parte.

Autores como Peattie e Peattie (2003), Gordon (2012), Parsons e Maclaran (2009) e Desai (2009) criticam a aplicação indiscriminada de princípios do marketing comercial em programas de marketing social e propõem mudanças que remetem à Lógica Dominada por Serviço. A principal crítica está na pouca atenção direcionada a fatores institucionais e sociais que influem no comportamento juntamente com os fatores de personalidade, diminuindo a efetividade dos programas. A redução no valor cocriado permitiria a ocorrência de recaídas para o comportamento indesejado anterior (PARSONS; MACLARAN, 2009).

As mudanças sugeridas são direcionadas principalmente ao mix de marketing, com novas propostas para a conceituação de produto, preço, praça e promoção. O conceito de produto, por exemplo, pode ser substituído pelo conceito de proposição de comportamento, dado que a organização realizadora do programa de marketing social não possui um pro- 
duto propriamente dito a ser oferecido no mercado. O produto em questão, que é o comportamento, seria coproduzido pelo público alvo e o valor cocriado (PEATTIE; PEATTIE, 2003).

Peattie e Peattie (2003) sugerem também a substituição do conceito de preço pelo de custo de envolvimento, que é determinado pelo beneficiário. Com relação à praça, a proposta é o conceito de acessibilidade, que incluiria tanto canais convencionais empregados na distribuição de produtos quanto canais que podem levar ao beneficiário a ideia de mudança, como interações com profissionais de saúde, professores e pais. Já o conceito de promoção deveria ser substituído pelo conceito de comunicação social, enfatizando o caráter de interação, envolvimento e compartilhamento de conhecimento (PEATTIE; PEATTIE, 2003).

Já Gordon (2012) propõe um mix de marketing social que coloca os atuais P's, como subcomponentes do componente canais/estratégias (meios de intervenção). Mais abrangente, o mix proposto pelo autor inclui também como componentes: as circunstâncias (ambiente social e estrutural, normas sociais); organização e competição (estrutura e relação entre stakeholders); custo (custo associado a mudança no comportamento); consumidor (avaliação e cocriação de valor); e processo (teoria, planejamento e estratégia orientada por valor).

Desai (2009), por sua vez, aborda os problemas causados pela aplicação do marketing de relacionamento tradicional e do gerenciamento da relação com o cliente no contexto do marketing social, como o tipo de relacionamento não perene entre programa social e beneficiários. Segundo o autor, os conceitos e práticas do relacionamento com o beneficiário baseadas na Lógica Dominada por Serviço podem ser aplicadas em programas de Marketing Social para aumentar o valor cocriado nas interações. As relações entre beneficiários e programas sociais se tornariam de parceria ativa de longo prazo, gerando maior valor de uso para todas as partes.

\subsection{Práticas de cocriação de valor e integração de recursos propostas pela SDL para o Marketing Social}

Um programa em Marketing Social funciona: (i) quando é baseado em pesquisa, planejamento, e modelos relevantes de mudança atitudinal e comportamental; (ii) quando todos os elementos do mix estão integrados; (iii) e quando os ambientes sócio-cultural, legislativo e estrutural facilitam ao público-alvo acesso ao programa (DONOVAN; HENLEY, 2010).

Do ponto de vista da Lógica Dominada por Serviço, um dos principais problemas em analisar os fenômenos em Marketing Social do ponto de vista da Lógica Dominada pelo Produto é a questão do valor do bem trocado e dos recursos integrados pelo público-alvo. Estes elementos não encontram respaldo teórico na Lógica Dominada pelo Produto, nem nas teorias econômicas clássicas, pois não há troca direta entre os atores (VARGO; LUSCH, 2017).

Os princípios do marketing comercial também não se mostraram muito apropriados para aplicações onde o produto ofertado é uma ideia e o resultado produzido pelo cliente, sendo alguns programas sociais proponentes de ações consideradas desagradáveis e inconvenientes pelos beneficiários (RUSSELL-BENNETT; PREVITE; ZAINUDDIN, 2009), como por exemplo, o uso de preservativo. Nesse caso, o valor econômico não é suficiente para analisar o fenômeno. A SDL oferece um conceito de valor multifacetado, que considera, além do valor de troca, o valor de uso e o valor em contexto (AKAKA; PARRY, 2019).

Ao incorporar aos aspectos econômicos os aspectos sociais da troca de serviço e o conceito de valor cocriado pela integração de recursos dos atores, a SDL oferece outra perspectiva de análise. Ao postular que o valor percebido pelo público-alvo dos programas em Marketing Social depende de aspectos sociais como instituições, símbolos, inseridos em uma lógica institucional, bem como de atividades ligadas a características psicológicas individuais e interações sociais, a SDL amplia o horizonte teórico da análise de fenômenos como a troca de serviço e a cocriação de valor.

No caso da cocriação de valor dentro da SDL, estudos de autores como McColl-Kennedy et al. (2012), 
Brambilla e Damacena (2012), Yi e Gong (2013), Akaka et al. (2014) e Neghina et al. (2015) revelaram como antecedentes do valor cocriado aspectos como a propensão a interações (ações recíprocas e coordenadas onde duas ou mais pessoas exercem efeito uma sobre as outras, com a oferta e demanda de colaboração) e tipos de atividades executadas pelos beneficiários (realizações ou esforços intrínsecos no sentido de buscar e compartilhar recursos, engajando-se de forma mais aguerrida ao processo da qual participa).
Em suma, após revisão bibliográfica nas pesquisas fundamentadas pela SDL sobre antecedentes da cocriação e valor, foi possível elaborar dois constructos (variáveis latentes não observáveis diretamente) para descrever a cocriação de valor: nível de atividades e número de interações. Esses constructos foram fundamentados com base nas escalas utilizadas pelos autores fonte, que por sua vez utilizaram as premissas da SDL relacionadas à cocriação de valor. Os itens que compõem cada constructo são mostrados no quadro 1 a seguir.

Quadro 1 itens que compõem os constructos sugeridos para o estudo e autores fonte.

\begin{tabular}{l|l|l}
\hline \multicolumn{1}{c|}{ Contructo } & \multicolumn{1}{c|}{ Item } & \multicolumn{1}{c}{ Autores fonte } \\
\hline \multirow{2}{*}{$\begin{array}{l}\text { Nível de } \\
\text { atividades - nat }\end{array}$} & $\begin{array}{l}\text { nat1- Acho importante sempre me manter informado com relação } \\
\text { a doenças sexualmente transmissíveis - DST e gravidez indesejada }\end{array}$ & McColl-Kennedy et al. (2012) \\
\cline { 2 - 3 } & $\begin{array}{l}\text { nat2- Normalmente procuro outras pessoas mais informadas quan- } \\
\text { do preciso resolver problemas relacionados às DST }\end{array}$ & McColl-Kennedy et al. (2012) \\
\cline { 2 - 4 } & $\begin{array}{l}\text { nat3- Acho importante compartilhar informações sobre como evi- } \\
\text { tar doenças sexualmente transmissíveis-DST e gravidez indesejada }\end{array}$ & Akaka et al. (2014), Neghina et al. (2015). \\
\hline \multirow{2}{*}{ inúmero de } & $\begin{array}{l}\text { ni1- Gosto de colaborar com as pessoas que tentam conscientizar } \\
\text { outros da importância do uso do preservativo }\end{array}$ & $\begin{array}{l}\text { Akaka et al. (2014), Brambilla e Damacena (2012), } \\
\text { Neghina et al. (2015). }\end{array}$ \\
\cline { 2 - 4 } & $\begin{array}{l}\text { ni2- Quando se trata de prevenir DST, acho importante ouvir o que } \\
\text { os outros dizem }\end{array}$ & McColl-Kennedy et al. (2012), \\
\cline { 2 - 3 } & $\begin{array}{l}\text { ni3- Quando o assunto é DST, quanto mais ajuda de outras pessoas } \\
\text { buscar, melhor }\end{array}$ & Yi e Gong (2013) \\
\hline
\end{tabular}

Fonte elaborado pelo autor baseado em McColl-Kennedy et al. (2012), Brambilla e Damacena (2012), Yi e Gong (2013), Akaka et al. (2014) e Neghina et al. (2015).

De acordo com os argumentos teóricos apresentados pelos autores presentes no quadro 1, pode-se afirmar que pessoas que apresentam comportamento com maior nível de atividades e número de interações tendem a cocriar valor maior, pois possuem e integram mais recursos. Mas, do ponto de vista do comportamento do consumidor (no caso beneficiário) dos programas sociais, quais antecedentes explicariam o nível de atividades e número de interações?

Uma possível resposta para essa pergunta foi encontrada na Psicologia, mais especificamente na Teoria do Traço de Personalidade e no Modelo Metateórico de Motivação e Personalidade - 3M (MOWEN, 2000) criado a partir dessa. O tópico a seguir expõe os princípios do modelo $3 \mathrm{M}$, o modelo hierárquico proposto e as hipóteses decorrentes dele.

\subsection{As bases teóricas do Modelo Metateórico de Motivação e Personalidade de Mowen (2000)}

Para o levantamento dos traços de personalidade que favoreçam a realização de atividades e interações utiliza-se o modelo $3 \mathrm{M}$ de motivação e comportamento (MOWEN, 2000). Esse modelo foi empregado em estudos de Avelar e Veiga (2013), investigando os antecedentes da vaidade feminina, e Ribeiro e Veiga (2011), que propuseram uma escala para mensuração de comportamento de consumo sustentável.

No modelo $3 \mathrm{M}$ o pressuposto fundamental postula que é possível integrar diversas abordagens de personalidade em uma meta-teoria unificada. A formulação do $3 \mathrm{M}$ é baseada na adaptação de escalas 
de personalidade existentes e na integração da psicologia evolucionária com a Teoria do Traço. Para Mowen (2000), os traços têm uma relação lógica com os componentes da teoria do controle presentes no modelo.

Um traço é visto como um constructo intra-psiquico que pode ser medido e prediz diferenças individuais em sentimentos, pensamentos e comportamentos. Desenvolvendo medidas válidas e confiáveis de traços, é possível identificar empiricamente relações entre comportamento, o contexto situacional, e as variáveis de personalidade. Os traços, no Modelo $3 \mathrm{M}$, são divididos em quatro níveis: elementares, compostos, situacionais e superficiais, ordenados hierarquicamente pelo nível de abstração dos mesmos.

Traços elementares, mais abstratos, resultam da genética e aprendizado na infância. Traços elementares se combinam com processos culturais e aprendizado na infância para o desenvolvimento de traços compostos. Traços compostos, por sua vez, combinam com o contexto do comportamento para criar traços situacionais (diferenças individuais expressas dentre de um contexto específico). Os traços situacionais interagem com atitudes e envolvimentos duradouros relacionados às classes de produtos criando traços superficiais (mais concretos e observáveis), ou tendências duradouras de agir com respeito a categorias de produtos ou domínio específico de comportamento (MOWEN, 2000).

Os traços elementares, que estão no nível mais abstrato, são fixos e sempre devem estar presentes no modelo de mensuração. Já os traços compostos, para serem considerados compostos, precisam ter pelo menos $25 \%$ de sua variação explicada por no mínimo dois traços elementares. São exemplos de traços compostos a autoeficácia, a competitividade, a orientação para tarefas e a necessidade de diversão. Traços compostos não fazem parte do modelo proposto, mas podem ser inseridos e, por meio de testes, ter sua influência verificada.

Os constructos sugeridos, nível de atividades e número de interações, são classificados como traços situacionais, pois expressam diferenças individuais no contexto do sexo seguro. Diferenças individuais em nível de atividades e número de interações determinam se as pessoas têm mais ou menos propensão a usar preservativos para garantir o sexo seguro, que no caso representaria o traço superficial (que pode ser observado no comportamento das pessoas por ser explícito).

O quadro 2 a seguir ilustra as definições dos traços elementares:

Quadro 2 Definição dos traços elementares do Modelo 3M

\begin{tabular}{l|l} 
Abertura a experiências (ae) & $\begin{array}{l}\text { Necessidade de encontrar novas soluções, expressar ideias originais e usar a imaginação na } \\
\text { execução de tarefas }\end{array}$ \\
\hline Organização (o) & Necessidade de ser organizado, ordenado e eficiente na realização de tarefas \\
\hline Introversão (i) & Tendência de vivenciar sentimentos de timidez \\
\hline Amabilidade (am) & Necessidade de expressar bondade e simpatia pelas pessoas \\
\hline Neuroticismo (n) & Tendência a expressar mal humor e atitude temperamental \\
\hline Necessidade de recursos materiais (nrm) & Necessidade de possuir e acumular bens materiais \\
\hline Necessidade de recursos corporais (nrc) & Necessidade de manter e realçar o corpo \\
\hline Necessidade de excitação (ne) & Desejo por estimulação e excitação \\
\hline
\end{tabular}

Fonte: Mowen (2000)

Os itens que mensuram os traços elementares foram definidos por Mowen (2000). Para que tenham sua validade e confiabilidade confirmada, os constructos sugeridos (que estão no nível situacional) precisam ter pelo menos $25 \%$ de sua variação expli- cada por pelo menos dois traços elementares, além de satisfazer os critérios de validade convergente, discriminante e confiabilidade composta. Caso determinado constructo apresente escala que não satisfaça os critérios de confiabilidade e unidimensionalidade, 
ele é excluído do modelo. No tópico 2 a seguir são explicitados o modelo e as hipóteses que dele derivam.

\section{O MODELO CONCEITUAL- HIPOTÉTICO E AS HIPÓTESES DA PESQUISA.}

Após pesquisa bibliográfica sobre os antecedentes da cocriação de valor, buscando ações e comportamentos que favorecem a adoção do uso frequente de preservativo (sexo seguro), foram elaborados dois constructos para traços situacionais: nível de atividades e número de interações. Em seguida, com o objetivo de levantar os traços elementares envolvidos com essas ações e comportamentos, criou-se um modelo conceitual-hipotético conforme terminologia aplicada na modelagem de equações estruturais.

Entre as várias ferramentas estatísticas disponíveis para a análise das relações entre constructos está a Modelagem de Equações Estruturais (MEE) ou Structural Equations Modeling (SEM). Segundo Hair Junior et al. (2005), a MEE permite avaliar a procedência estatística, a magnitude e a direção dos diversos caminhos causais de modelos. Dentro da SEM, os modelos são formados por variáveis latentes e variáveis observadas (ou manifestas) (KLINE, 2011)"publisher":"The Guilford Press"',publisher-place":"New York'",'edition":"3'”,'event-place":"New

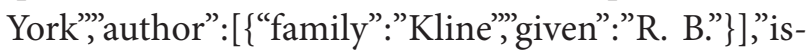
sued":\{“date-parts":[[“2011”]]\}\}\}],"schema”:”https:// github.com/citation-style-language/schema/raw/ master/csl-citation.json"\} .

As variáveis latentes equivalem aos constructos citados por Selltiz et al. (1987), que podem ser de dois tipos: constructos endógenos e exógenos. Os primeiros equivalem a uma variável dependente, ou seja, aquela que em um diagrama possui uma ou mais setas indicadas para ela. Já os constructos exógenos são aqueles equivalentes às variáveis independentes (HAIR JUNIOR et al., 2005).

Quanto às variáveis observadas, estas, como citado por Selltiz et al. (1987), são aquelas que representam o constructo de maneira concreta, e são coletadas por instrumentos como questionários, e tabuladas em arquivos de dados (KLINE, 2011). A Modelagem de Equações Estruturais, segundo Hair
Junior et al. (2009, p.627) "viabiliza uma forma conceitualmente atraente de testar uma teoria".

O modelo estrutural hierárquico, mostrado na figura 1, mostra os constructos e as relações entre eles. As setas indicam os caminhos ou relações de dependência entre os constructos. No caso, os traços elementares formam o conjunto de constructos exógenos (independentes) e os traços situacionais (nível de atividade e número de interações) os constructos endógenos (dependentes).

Figura 1 modelo estrutural hierárquico para mensuração dos antecedentes dos constructos sugeridos

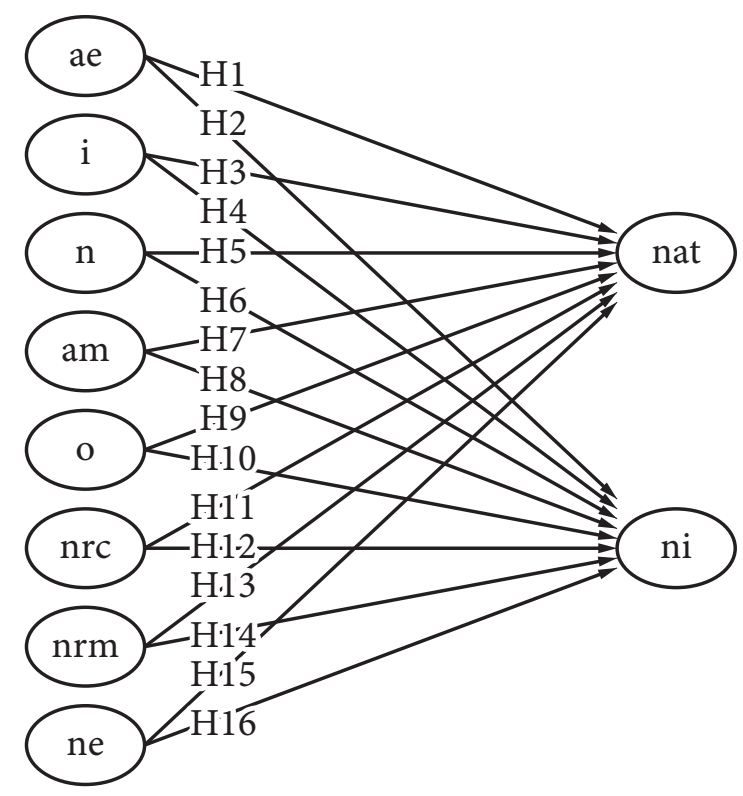

Nota ae=abertura a experiências, $\mathrm{i}=$ introversão, $\mathrm{n}=$ neuroticismo, am=amabilidade, o=organização, $\mathrm{nrc}=$ necessidade de recursos corporais, $\mathrm{nrm}=$ necessidade de recursos materiais, ne=necessidade de excitação, nat=nível de atividades, ni=número de interações.

Fonte elaborado pelo autor, 2017

A partir do modelo são identificadas as hipóteses de pesquisa. $\mathrm{O}$ modelo pode ser considerado parcialmente mediado, pois sugere que há relações entre todos os constructos envolvidos. Devido ao caráter exploratório do modelo, os constructos nível de atividades e número de interações ainda não foram analisados por estudos anteriores. Por isso as relações entre eles e os traços elementares foram levantados por dedução lógica.

Considerando que os traços situacionais sugeridos contemplam aspectos como iniciativa pessoal na busca de informações, propensão a interagir e 
colaborar, e propensão ao engajamento, as hipóteses de relações entre os constructos são levantadas de acordo com o alinhamento entre o traço elementar e os aspectos citados. Por exemplo, espera-se que pessoas com abertura a experiência apresentem iniciativa e propensão a interagir na busca por novos modos de realizar ações. Assim, as hipóteses do modelo proposto são:

- H1: abertura a experiência está positivamente relacionado a nível de atividades

- H2- abertura a experiência está positivamente relacionado a número de interações

- H3- introversão está negativamente relacionado a nível de atividades

- H4- introversão está negativamente relacionado a número de interações

- H5- neuroticismo está positivamente relacionado a nível de atividades

- H6- neuroticismo está positivamente relacionado a número de interações

- H7- amabilidade está positivamente relacionado a nível de atividades

- H8- amabilidade está positivamente relacionado a número de interações

- H9- organização está positivamente relacionado a nível de atividades

- H10- organização está positivamente relacionado a número de interações

- H11- necessidade de recursos materiais está negativamente relacionado a nível de atividades

- H12-necessidade de recursos materiais está negativamente relacionado a número de interações

- H13- necessidade de recursos corporais está positivamente relacionado a nível de atividades

- H14- necessidade de recursos corporais está positivamente relacionado a número de interações

- H15- necessidade de excitação está positivamente relacionado a nível de atividades

- H16- necessidade de excitação está positivamente relacionado a número de interações

A seguir são expostos os procedimentos utilizados para verificar a confiabilidade, unidimensionalidade, confiabilidade composta, validade discriminante, validade convergente e grau da qualidade de ajuste do modelo proposto.

\section{MÉTODO}

Esse trabalho pode ser classificado, com relação ao objetivo, como descritivo, pois objetiva descrever as características de um fenômeno, por meio do estabelecimento de relações entre variáveis (GIL, 2006). Com relação ao tipo de abordagem, trata-se de um estudo quantitativo onde as variáveis são quantificadas em números para posterior análise estatística (VERGARA, 2005). O instrumento de coleta de dados utilizado foi o questionário, aplicado por entrevistador treinado para amenizar possíveis constrangimentos aos respondentes em decorrência do tema íntimo da pesquisa.

Os questionários foram aplicados a uma amostra por conveniência de alunos do curso de Administração da Universidade Federal dos Vales do Jequitinhonha e Mucuri, no período de 25 de janeiro a 08 de fevereiro de 2017, como parte de trabalho de avaliação de disciplina do curso voltada para a pesquisa de marketing. Após a coleta os questionários foram depurados e os que apresentavam respostas duplas, omissas e rasuras foram descartados. Ao final da coleta e depuração foram tabulados 160 questionários.

Após a tabulação, em planilha do software SPSS ${ }^{\circledast}$, primeiramente foram feitas buscas por casos considerados outliers univariados e multivariados. Para os outliers univariados foi adotado o critério de Tukey (1977), onde casos com valores fora do intervalo Q1-1,5IQR - Q3+1,5IQR são considerados outliers e excluídos da análise. Para a detecção de outliers multivariados foi utilizado o critério da distância de Mahalanobis (MINGOTI, 2005).

Após o tratamento dos casos com valores outliers foram feitos os testes de unidimensionalidade das escalas dos constructos por meio de análise fatorial exploratória (exigência: $\mathrm{KMO}>0,6$, variância explicada pelo fator $>60 \%$ ). Após o teste de unidimensionalidade foi feito o teste de confiabilidade (consistência interna) utilizando o índice alpha de Cronbach (exigência: alpha $\geq 0,6$ ).

Em seguida, utilizando-se o software $\mathrm{AMOS}^{\circledR} 5$ foram feitos os testes de validade convergente, validade discriminante e confiabilidade composta. Para essa avaliação utilizou-se a análise fatorial confirmatória feita no modelo de mensuração, onde foram calculadas as covariâncias entre os constructos. 
Da análise foi calculada a média de variância extraída (AVE) e a confiabilidade composta (CR) para verificação da validade convergente (exigência: $A V E \geq 0,45$ e $C R \geq 0,6$ ). Para verificação da validade discriminante foram comparados os valores da raiz quadrada de AVE com as correlações entre fatores (exigência: correlação $<\sqrt{\mathrm{AVE}}$ ) (FORNELL; LARCKER, 1981).

Uma vez confirmadas as validades convergente, discriminante e confiabilidade composta, efetuou-se o teste de qualidade ajuste do modelo e a verificação das hipóteses aceitas. $\mathrm{O}$ software $\mathrm{AMOS}^{\circledR} 5$ forneceu os valores de $\chi^{2}, \chi^{2} / \mathrm{df}$, CFI (Comparative Fit index) e RMSEA (Root Mean Square Error of Aproximation) para verificação da qualidade de ajuste do modelo (exigências: $\chi^{2}$ significativo, $\chi 2 / \mathrm{df}<5, \mathrm{CFI} \geq 0,90$, RMSEA $<0,08)$

\section{ANÁLISE DOS RESULTADOS}

\subsection{Outliers univariados}

Com relação aos outliers univariados, não foram detectados valores fora do intervalo determinado nas respostas dos questionários para cada constructo.

\subsection{Outliers multivariados}

Com relação aos outliers multivariados, também não foram encontrados casos onde a distância de Mahalanobis fosse significante $(\mathrm{p}<0,001)$.

\subsection{Teste de unidimensionalidade das escalas}

A tabela 1 a seguir ilustra os resultados da análise fatorial exploratória realizada individualmente com cada constructo do modelo proposto.

Tabela 1 Resultados da análise fatorial exploratória

\begin{tabular}{|c|c|c|c|c|c|c|}
\hline & Constructo & kmo & barlett & no fatores & $\begin{array}{l}\text { variância } \\
\text { explicada }\end{array}$ & Comentário \\
\hline 1 & abertura experiência & 0,652 & 0 & 1 & 66,6 & \\
\hline 2 & introversão & 0,6 & 0 & 1 & 60 & exclui i1 \\
\hline 3 & neuroticismo & 0,823 & 0 & 1 & 60,21 & \\
\hline 4 & amabilidade & 0,722 & 0 & 1 & 74,76 & \\
\hline 5 & organização & 0,637 & 0 & 1 & 58,14 & exclui o3 \\
\hline 6 & nec. rec. corporais & 0,648 & 0 & 1 & 60,59 & exclui nrc4 \\
\hline 7 & nec. rec.materiais & 0,692 & 0 & 1 & 73,28 & exclui nrm1 \\
\hline 8 & nec. excitação & 0,611 & 0 & 1 & 69,26 & exclui ne3 \\
\hline 9 & nivel de atividade & 0,651 & 0 & 1 & 72,18 & \\
\hline 10 & número de interações & 0,658 & 0 & 1 & 70,57 & \\
\hline
\end{tabular}

Fonte elaborado pelo autor, 2017

Após execução da análise fatorial exploratória, verificou-se que os constructos dos traços elementares introversão, organização, necessidade de recursos corporais, necessidade de recursos materiais e necessidade de excitação só atenderiam aos requisitos mínimos de unidimensionalidade se fossem excluídos um dos itens da escala.
O critério de exclusão foi a presença de carga fatorial significativa em mais de um fator. No caso do constructo organização, mesmo com a exclusão do item 03 (faço minhas atividades de forma sistemática), a variância explicada pelo fator ficou abaixo de $60 \%$. Como o valor de KMO ficou acima do mínimo de 0,6 , decidiu por manter o constructo na análise. 


\subsection{4 Teste de consistência interna das escalas}

Para o teste de consistência interna (confiabilidade) foi feito o teste alpha de Cronbach, já sem os itens excluídos no teste de unidimensionalidade. Os resultados são mostrados na tabela 2 .

Os resultados mostram que todos os constructos apresentaram valores de alpha de Cronbach acima do mínimo de 0,6 , mostrando que as escalas possuem consistência interna.

\subsection{Validade convergente, confiabilidade composta e validade discriminante dos constructos}

A análise fatorial confirmatória, realizada no software $\mathrm{AMOS}^{\circledR} 5$ calculando as correlações entre todos os constructos apresentou, como ilustrado na tabela 3, os seguintes resultados de AVE, confiabilidade composta (CR) e $\sqrt{\mathrm{AVE}}$.
Tabela 2 Resultados do teste alpha de Cronbach

\begin{tabular}{c|c|c}
\hline & Constructo & alpha \\
\hline 1 & abertura experiência & 0,744 \\
\hline 2 & introversão & 0,663 \\
\hline 3 & neuroticismo & 0,83 \\
\hline 4 & amabilidade & 0,828 \\
\hline 5 & organização & 0,624 \\
\hline 6 & nec rec corporais & 0,669 \\
\hline 7 & nec rec materiais & 0,816 \\
\hline 8 & nec excitação & 0,77 \\
\hline 9 & nivel de atividade & 0,805 \\
\hline 10 & número de interações & 0,773 \\
\hline
\end{tabular}

Fonte elaborado pelo autor, 2017

Tabela 3 Valores de AVE, CR, $\sqrt{\mathrm{AVE}}$ e correlação entre os constructos do modelo

\begin{tabular}{c|c|c|c|c|c|c|c|c|c|c|c|c}
\hline & $\mathbf{C R}$ & $\mathbf{A V E}$ & $\mathbf{i}$ & $\mathbf{a e}$ & $\mathbf{n}$ & $\mathbf{a m}$ & $\mathbf{0}$ & $\mathbf{n r c}$ & $\mathbf{n r m}$ & $\mathbf{n e}$ & $\mathbf{n a t}$ & $\mathbf{n i}$ \\
\hline $\mathbf{i}$ & 0,681 & 0,367 & $\mathbf{0 , 6 0 6}$ & & & & & & & & & \\
\hline $\mathbf{a e}$ & 0,759 & 0,517 & $-0,161$ & $\mathbf{0 , 7 1 9}$ & & & & & & & & \\
\hline $\mathbf{n}$ & 0,831 & 0,502 & 0,245 & $-0,115$ & $\mathbf{0 , 7 0 9}$ & & & & & & & \\
\hline $\mathbf{a m}$ & 0,83 & 0,62 & 0,046 & 0,223 & $-0,514$ & $\mathbf{0 , 7 8 8}$ & & & & & & \\
\hline $\mathbf{0}$ & 0,631 & 0,314 & 0,155 & 0,441 & $-0,468$ & 0,546 & $\mathbf{0 , 5 6}$ & & & & & \\
\hline $\mathbf{n r c}$ & 0,709 & 0,38 & $-0,005$ & 0,386 & $-0,128$ & 0,229 & 0,152 & $\mathbf{0 , 6 1 6}$ & & & & \\
\hline $\mathbf{n r m}$ & 0,743 & 0,463 & $-0,1$ & 0,147 & 0,366 & $-0,178$ & $-0,161$ & 0,359 & $\mathbf{0 , 6 8}$ & & & \\
\hline $\mathbf{n e}$ & 0,763 & 0,477 & $-0,044$ & 0,108 & 0,151 & $-0,094$ & $-0,154$ & 0,193 & 0,436 & $\mathbf{0 , 6 9}$ & & \\
\hline nat & 0,818 & 0,602 & 0,195 & 0,323 & 0,008 & 0,105 & 0,235 & 0,202 & 0,016 & 0,042 & $\mathbf{0 , 7 7 6}$ & \\
\hline $\mathbf{n i}$ & 0,8 & 0,574 & 0,131 & 0,264 & $-0,016$ & 0,195 & 0,293 & $-0,061$ & $-0,105$ & $-0,095$ & 0,843 & $\mathbf{0 , 7 5 7}$ \\
\hline
\end{tabular}

Nota ae=abertura a experiências, $\mathrm{i}=$ introversão, $\mathrm{n}=$ neuroticismo, am=amabilidade, $\mathrm{o}=$ organização, $\mathrm{nrc}=$ necessidade de recursos corporais, $\mathrm{nrm}=$ necessidade de recursos materiais, ne=necessidade de excitação, nat=nível de atividades, ni=número de interações. Números em negrito na diagonal representam $\sqrt{\mathrm{AVE}}$.

Fonte elaborado pelo autor, 2017

O modelo de mensuração apresentou os seguintes índices de qualidade de ajuste: $\chi 2=966,901$, $\mathrm{df}=584, \mathrm{p}<0,01, \chi^{2} / \mathrm{df}=1,656, \mathrm{CFI}=0,822$, $\mathrm{RMSEA}=0,064$. Os valores em negrito na diagonal representam $\sqrt{\mathrm{AVE}}$, e são comparados com os va- lores abaixo na coluna para verificação de validade discriminante. Os valores de ajuste mostram que o modelo apresenta ajuste marginalmente satisfatório, pois o valor de CFI ficou abaixo de 0,90 . 
Todos os constructos apresentaram confiabilidade composta satisfatória, com valores acima de 0,6. Já com relação à validade convergente, os constructos introversão, organização e necessidade de recursos corporais apresentaram AVE abaixo do mínimo de 0,45 e por isso foram excluídos do modelo.

O principal problema ficou por conta da falta de validade discriminante apresentada entre os dois constructos sugeridos no estudo. Como o valor da correlação $(0,843)$ é superior à raiz da variância média extraída $(0,776)$, não se pode afirmar que estes dois constructos medem apenas o que se propõem a medir. Entretanto, utilizando um critério menos rigoroso, se a correlação entre dois constructos for menor que 0,85 , pode-se considerar, embora de maneira menos robusta, que esses possuem validade discriminante entre si (NETEMEYER; BEARDEN; SHARMA, 2003).

Com a exclusão dos constructos citados, o novo modelo de mensuração apresentou os seguintes valores de qualidade de ajuste: $\chi 2=410,957, \mathrm{df}=254$, $\mathrm{p}<0,01, \chi 2 / \mathrm{df}=1,617, \mathrm{CFI}=0,90, \mathrm{RMSEA}=0,062$. Observa-se que a qualidade de ajuste apresentou melhoras (CFI passou de 0,822 para 0,90, atingindo o mínimo desejado). Fazendo nova análise de validade convergente, discriminante e de confiabilidade composta no modelo de mensuração sem os constructos excluídos, obteve-se os resultados mostrados na tabela 4 a seguir:

Tabela 4 Valores de AVE, CR, $\sqrt{\mathrm{AVE}}$ e correlação entre os constructos do modelo corrigido

\begin{tabular}{c|c|c|c|c|c|c|c|c|c}
\hline & CR & AVE & ni & ae & n & am & nrm & ne & nat \\
\hline ni & 0,801 & 0,574 & $\mathbf{0 , 7 5 8}$ & & & & & & \\
\hline ae & 0,755 & 0,512 & 0,280 & $\mathbf{0 , 7 1 5}$ & & & & & \\
\hline $\mathbf{n}$ & 0,831 & 0,502 & $-0,014$ & $-0,125$ & $\mathbf{0 , 7 0 9}$ & & & & \\
\hline am & 0,830 & 0,620 & 0,195 & 0,240 & $-0,509$ & $\mathbf{0 , 7 8 8}$ & & & \\
\hline nrm & 0,742 & 0,462 & $-0,105$ & 0,150 & 0,367 & $-0,179$ & $\mathbf{0 , 6 8 0}$ & & \\
\hline ne & 0,763 & 0,477 & $-0,093$ & 0,115 & 0,152 & $-0,093$ & 0,437 & $\mathbf{0 , 6 9 1}$ & \\
\hline nat & 0,817 & 0,600 & 0,848 & 0,350 & 0,009 & 0,106 & 0,012 & 0,041 & $\mathbf{0 , 7 7 5}$ \\
\hline
\end{tabular}

Nota $a e=$ abertura a experiências, $\mathrm{n}=$ neuroticismo, $\mathrm{am}=$ amabilidade, $\mathrm{nrm}=$ necessidade de recursos materiais, $\mathrm{ne}=\mathrm{necessidade}$ de excitação, nat = nível de atividades, ni = número de interações. Números em negrito na diagonal representam $\sqrt{\mathrm{AVE}}$.

Fonte elaborado pelo autor, 2017.

Como pode-se verificar, no modelo de mensuração reduzido, todos os constructos apresentam validade convergente, discriminante e confiabilidade composta com valores satisfatórios (AVE $\geq 0,45$ e $\mathrm{CR} \geq 0,6$, correlação com outros constructos $<$ com exceção dos constructos nat e ni).

\subsection{Qualidade de ajuste do modelo estrutural}

Com a exclusão dos constructos que não apresentaram validade convergente, e dos itens que apresentaram carga significativa em mais de um fator (nrm1 e ne3), o novo modelo estrutural ficou da seguinte forma:
Figura 2 modelo estrutural hierárquico reduzido

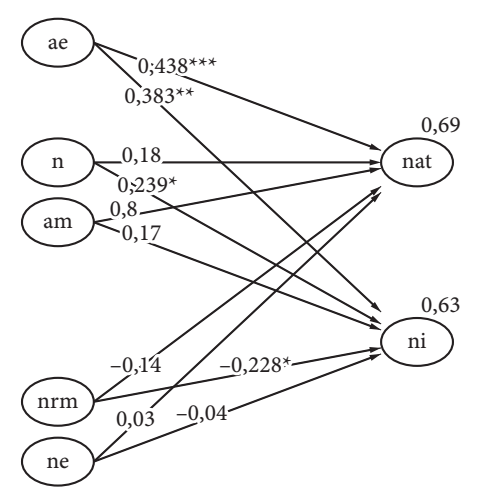

Nota ae $=$ abertura a experiências, $\mathrm{n}=$ neuroticismo, $\mathrm{am}=$ amabilidade, $\mathrm{nrm}=$ necessidade de recursos materiais, $\mathrm{ne}=$ necessidade de excitação, nat $=$ nível de atividades, $\mathrm{ni}=$ número de interações. *** $=p<0,001,{ }^{* *}=p<0,05,{ }^{*}=p<0,1$.

Fonte elaborado pelo autor, 2017. 
Os índices de qualidade de ajuste apresentaram os seguintes valores: $\chi^{2}=433,699, \mathrm{df}=210, \mathrm{p}<0,01$, $\chi^{2 / \mathrm{df}}=2,065, \mathrm{CFI}=0,854$, RMSEA $=0,082$, indicando ajuste marginalmente satisfatório. Pelos resultados apresentados no modelo, pode-se verificar que o $\mathrm{R}^{2}$ para o constructo nível de atividades (nat) foi de 0,69 ou $69 \%$. Para o constructo número de interações o $\mathrm{R}^{2}$ foi 0,63 ou $63 \%$. Esses resultados mostram que parte considerável da variância dos constructos sugeridos é explicada pelos constructos de traços elementares. Para levantar quais traços tem maior peso, analisam-se as estimativas de efeitos entre os constructos no modelo hierárquico reduzido, que são mostrados na tabela 5 .

Tabela 5 Estimativas de efeitos entre os constructos e variância explicada

\begin{tabular}{|c|c|c|c|c|c|c|c|c|c|}
\hline & Relação & & $\begin{array}{l}\text { Estima- } \\
\text { tiva }\end{array}$ & S.E. & C.R. & $\begin{array}{c}\text { Estimati- } \\
\text { va Padro- } \\
\text { nizada }\end{array}$ & $\mathbf{p}$ & sig. & $\mathbf{R}^{2}$ \\
\hline nat & $\leftarrow$ & ae & 0,654 & 0,184 & 3,563 & 0,438 & 0,000 & $* * *$ & \multirow{5}{*}{$69 \%$} \\
\hline nat & $\leftarrow$ & $\mathrm{nrm}$ & $-0,16$ & 0,128 & $-1,253$ & $-0,14$ & 0,21 & NS & \\
\hline nat & $\leftarrow$ & ne & 0,047 & 0,159 & 0,294 & 0,028 & 0,769 & NS & \\
\hline nat & $\leftarrow$ & $n$ & 0,266 & 0,173 & 1,536 & 0,183 & 0,125 & NS & \\
\hline nat & $\leftarrow$ & am & 0,11 & 0,152 & 0,725 & 0,081 & 0,468 & NS & \\
\hline ni & $\leftarrow$ & ae & 0,565 & 0,178 & 3,173 & 0,383 & 0,002 & $* *$ & \multirow{5}{*}{$63 \%$} \\
\hline ni & $\leftarrow$ & $\mathrm{nrm}$ & $-0,256$ & 0,132 & $-1,944$ & $-0,228$ & 0,052 & * & \\
\hline ni & $\leftarrow$ & ne & $-0,059$ & 0,16 & $-0,367$ & $-0,036$ & 0,713 & NS & \\
\hline ni & $\leftarrow$ & $n$ & 0,341 & 0,179 & 1,903 & 0,239 & 0,057 & * & \\
\hline ni & $\leftarrow$ & am & 0,221 & 0,155 & 1,428 & 0,165 & 0,153 & NS & \\
\hline
\end{tabular}

Nota ae=abertura a experiências, $\mathrm{n}=$ neuroticismo, am=amabilidade, $\mathrm{nrm}=$ necessidade de recursos materiais, ne=necessidade de excitação, nat=nível de atividades, ni=número de interações.

$* * *=p<0,001,{ }^{* *}=p<0,05,{ }^{*}=p<0,1, N S=$ não significativo.

Fonte elaborado pelo autor, 2017.

Quadro 3 Resultados dos testes de hipóteses

\begin{tabular}{|c|c|c|}
\hline Hipótese & Relação & Resultado \\
\hline $\mathrm{H} 1$ & abertura a experiência está positivamente relacionado a nível de atividades & suportada \\
\hline $\mathrm{H} 2$ & abertura a experiência está positivamente relacionado a número de interações & suportada \\
\hline $\mathrm{H} 5$ & neuroticismo está positivamente relacionado a nível de atividades & Não suportada \\
\hline H6 & neuroticismo está positivamente relacionado a número de interações & suportada \\
\hline $\mathrm{H7}$ & amabilidade está positivamente relacionado a nível de atividades & Não suportada \\
\hline H8 & amabilidade está positivamente relacionado a número de interações & Não suportada \\
\hline $\mathrm{H} 11$ & necessidade de recursos materiais está negativamente relacionado a nível de atividades & Não suportada \\
\hline $\mathrm{H} 12$ & necessidade de recursos materiais está negativamente relacionado a número de interações & suportada \\
\hline $\mathrm{H} 15$ & necessidade de excitação está positivamente relacionado a nível de atividades & Não suportada \\
\hline $\mathrm{H} 16$ & necessidade de excitação está positivamente relacionado a número de interações & Não suportada \\
\hline
\end{tabular}

Fonte elaborado pelo autor, 2017. 
Pelos resultados percebe-se que o nível de atividades (nat) é explicado somente pelo traço elementar abertura a experiência $(0,438, p<0,01)$. Já para o construto número de interações (ni), os traços que o explicam são abertura a experiência $(0,383, p<0,05)$, neuroticismo $(0,239, p<0,1)$, e necessidade de recursos materiais $(-0,228, p<0,1)$.

\subsection{Teste de hipóteses}

Com os resultados da tabela 5 é possível também efetuar o teste das hipóteses presentes no modelo reduzido. Para que uma hipótese seja suportada é necessário que a estimativa de efeito entre os constructos seja significativa, ou seja, seja menor que 0,1 . $\mathrm{O}$ resultado do teste das hipóteses remanescentes após depuração do modelo é mostrado no quadro 3:

Os resultados do teste de hipóteses indica que o traço elementar abertura à experiência é um antecedente tanto do traço nível de atividades, quanto do traço número de interações. O traço neuroticismo é antecedente do traço número de interações, assim como o traço necessidade de recursos materiais. Esses traços elementares seriam, então, os antecedentes da cocriação de valor no contexto estudado.

\section{CONSIDERAÇÕES FINAIS}

Esse estudo analisou um fenômeno da área do Marketing Social, empregando arcabouço teórico da Lógica Dominada por Serviço (VARGO; LUSCH, 2016), tendo como teoria de médio alcance para servir de ponte entre a teoria mais geral (SDL) e o contexto empírico (BRODIE; SAREN; PELS, 2011) o Modelo Metateórico de Motivação e Personalidade (MOWEN, 2000).

O objetivo foi propor e validar um modelo de cocriação de valor baseado no corpo teórico da Lógica Dominada por Serviço, contendo constructos envolvidos no processo de cocriação de valor (traços de personalidade compostos) e seus possíveis antecedentes (traços de personalidade elementares). Após pesquisa bibliográfica elaborou-se os constructos e seus respectivos itens de escala, e como possíveis antecedentes foram adotados os traços elementares do modelo 3M de Mowen (2000), utilizados fre- quentemente em estudos sobre comportamento do consumidor.

Após aplicação empírica em estudantes do curso de Administração da Universidade Federal dos Vales do Jequitinhonha e Mucuri, os resultados da aplicação da ferramenta proposta revelaram que o constructo nível de atividades possui como antecedente o traço elementar abertura a experiência. Daí pode-se inferir que, se o uso representar uma experiência nova para o beneficiário, a adoção é mais provável. Já o constructo número de interações possui como antecedentes os traços elementares abertura a experiência, neuroticismo e necessidades de recursos materiais (negativamente).

Dos resultados infere-se que é possível maximizar o valor cocriado pelos estudantes, com relação ao sexo seguro, fazendo com que estes adotem comportamentos perenes que levem a maior busca e compartilhamento de informações e engajamento no ativismo para sexo seguro, e também a maior número de interações, ajudando e sendo ajudado nas situações onde o uso de preservativo é importante.

Essa informação pode ser utilizada pelos gestores de programas sociais para nortear ações que incentivam o uso frequente de preservativo entre jovens da região do Vale do Mucuri, oferecendo aos beneficiários recursos que possam ser integrados de maneira coordenada com os recursos de outros atores (poder público, famílias, escolas, e comunidades) e permitam a mudança voluntária de comportamento, e a redução nos índices de ocorrência de doenças sexualmente transmissíveis e gravidez indesejada.

\subsection{Limitações da pesquisa}

Essa pesquisa apresentou várias limitações. No momento da coleta de dados, há sempre a possibilidade da opção por respostas socialmente desejáveis. A amostragem por conveniência torna o estudo não probabilístico, por isso os resultados alcançados são aplicáveis apenas nessa amostra de alunos. Na análise estatística, é aconselhável ter pelo menos 5 casos para cada item das escalas do questionário, o que exigiria pelo menos 185 questionários respondidos. Porém conseguiu-se apenas 160 respostas. Há as limitações decorrentes da falta de normalidade dos dados, prérequisito para algumas análises efetuadas. 
Tais limitações podem ter culminado na validade discriminante marginalmente satisfatória apresentada entre os dois constructos sugeridos (nível de atividades e número de interações) no teste AVE, que mostra que os itens dessas escalas precisam de refinamento.

\subsection{Sugestão de temas para continuidade do estudo}

Entre as sugestões de estudo estão a análise da influência de traços compostos como a autoeficácia e necessidade de aprendizado, que por dedução lógica, apresentam relação com os traços situacionais sugeridos nesse estudo.

\section{BIBLIOGRAFIA}

AKAKA, M. A.; PARRY, G. Value-in-Context: An Exploration of the Context of Value and the Value of Context. In: MAGLIO, P. P. et al. (Org.). . Handbook of Service Science. Switzerland: Springer Nature, 2019. v. II.

AKAKA, M. A. et al. The role of symbols in value cocreation. Marketing Theory, v. 14, n. 3, p. 311-326, 1 set. 2014.

ANDREASEN, A. R. Marketing Social Marketing in the Social Change Marketplace. Journal of Public Policy \& Marketing, v. 21, n. 1, p. 3-13, 2002.

ANDREASEN, A. R. Social Marketing: Its definition and Domain. Journal of Public Policy \& Marketing, v. 13, n. 01, 1994.

BARON, S. et al. Service-dominant logic: marketing research implications and opportunities. Journal of Consumer Behavior, v. 9, n. 3, p. 253-264, 2010.

BEALL, T. et al. Social marketing at a critical turning point. Journal of Social Marketing, v. 2, n. 2, p. 103-117, 2012.
BETTENCOURT, L. A.; LUSCH, R. F.; VARGO, S. L. A service lens on value creation: marketing's role in achieving strategic advantage. California Management Review, v. 57, n. 1, p. 44-66, 2014.

BRAMBILLA, F. R.; DAMACENA, C. Estudo etnometodológico da cocriação de valor no ensino superior privado de administração com base na lógica dominante do serviço em marketing. Revista Brasileira de Marketing, v. 11, n. 3, p. 124-153, 2012.

BRODIE, R. J.; SAREN, M.; PELS, J. Theorizing about the service dominant logic: The bridging role of middle range theory. Marketing Theory, v. 11, n. 1, p. 75-91, 2011.

CALERO-GIMENO, R.; GALLARZA-GRANIZO, M. G. Aplicabilidad del Service-Dominant Logic al ámbito sanitario: caracterizando el servicio para la co-creación de valor. Rev. Gerenc. Polit. Salud, v. 14, n. 29, p. 179-192, 2015.

DESAI, D. Role of Relationship Management and Value Co-Creation in Social Marketing. Social Marketing Quarterly, v. XV, n. 4, p. 112-125, 2009.

FORNELL, C.; LARCKER, D. F. Evaluating Structural Equations Models with Unobservable Variables and Measurement Errors. Journal of Marketing Research, v. 18, n. 1, p. 39-50, 1981.

GIL, A. C. Métodos e Técnicas de Pesquisa Social. 5. ed. São Paulo: Atlas, 2006.

GORDON, R. Re-thinking and re-tooling the social marketing mix. Australasian Marketing Journal, v. 20, n. 2, p. 122-126, 2012.

HAIR JUNIOR, J. F. et al. Análise multivariada de dados. 5. ed. Porto Alegre: Bookman, 2005.

HIETANEN, J.; ANDÉHN, M.; BRADSHAW, A. Against the implicit politics of service-dominant logic. Marketing Theory, v. 18, n. 1, p. 101-119, 2018. 
HUNT, S. Foundations of Marketing Theory: toward a general theory of marketing. new york: M. E. Sharpe, 2002.

KLINE, R. B. Principles and practice of structural equation modeling. 3. ed. New York: The Guilford Press, 2011.

KOTLER, P.; KELLER, K. L. Administração de Marketing para o Século XXI: como criar, conquistar e dominar mercados. São Paulo: Pearson, 2012.

LEVITT, T. A imaginação de Marketing. São Paulo: Atlas, 1985.

LUCA, N. R.; HIBBERT, S.; MCDONALD, R. Towards a service dominant approach to social marketing. Marketing Theory, p. 1-25, 2015.

LUSCH, R. F.; VARGO, S. L. The Service-dominant Logic of Marketing: Dialog, Debate, and Directions. [S.l.]: M.E. Sharpe, 2006.

LUSCH, R. L.; VARGO, S. L. Service-Dominant Logic: premises, perspectives, possibilities. Cambridge: Cambridge University Press, 2014.

MCCOLL-KENNEDY, J. R. et al. Health Care Costumer Value Cocreation Practice Styles. Journal of Service Research, v. 15, n. 4, p. 370-389, 2012.

MINGOTI, S. A. Análise de dados através de métodos de estatística multivariada: uma abordagem aplicada. Belo Horizonte: Editora UFMG, 2005.

MOWEN, J. C. The $3 M$ model of motivation and personality: theory and empirical applications to consumer behavior. New York: Springer Science+Business Media, 2000.

NEGHINA, C. et al. Value cocreation in service interactions: Dimensions and antecedents. Marketing Theory, v. 15, n. 2, p. 221-242, 2015.

NETEMEYER, R. G.; BEARDEN, W. O.; SHARMA, S. Scaling procedures: Issues and applications. [S.l.]: Sage Publications, 2003.
PARSONS, E.; MACLARAN, P. Contemporary Issues in Marketing and Consumer Behaviour. Oxford: Elsevier, 2009.

PAYNE, A. F.; STORBACKA, K.; FROW, P. Managing the co-creation of value. Journal of the Academy of Marketing Science, v. 36, p. 83-96, 2008.

PEATTIE, S.; PEATTIE, K. Ready to fly solo? Reducing social marketing's dependence on commercial marketing theory. Marketing Theory, v. 3, n. 3, p. 365-385, 2003.

PETERS, L. D. et al. Theorizing about resource integration through service-dominant logic. Marketing Theory, v. 14, n. 3, p. 249-268, 1 set. 2014.

POHLMANN, A.; KAARTEMO, V. Research trajectories of Service-Dominant Logic: Emergent themes of a unifying paradigm in business and management. Industrial Marketing Management, v. 63, p. 53-68, 2017.

PRAHALAD, C. K.; RAMASWAMY, V. Co-creation experiences: the next practice in value creation. Journal of Interactive Marketing, v. 18, n. 3, 2004.

RUSSELL-BENNETT, R.; PREVITE, J.; ZAINUDDIN, $\mathrm{N}$. Conceptualising value creation for social change management. Australasian Marketing Journal, v. 17, n. 4, p. 211-218, 2009.

SELLTIZ, C.; WRIGHTSMAN, L. S.; COOK, S. W. Métodos de pesquisa nas relações sociais. 11. ed. São Paulo: EPU, 1987. v. II.

TUKEY, J. W. Exploratory data analysis. [S.1.]: Addison-Wesley, 1977.

VARGO, S. L.; LUSCH, R. F. Institutions and axioms: an extension and update of service-dominant logic. Journal of the Academy Marketing Science, 00054, v. 44, p. 5-23, 2016.

VARGO, S. L.; LUSCH, R. F. Service-dominant logic 2025. International Journal of Research in Marketing, v. 34, p. 45-67, 2017. 
VEIGA, R. T. Fundamentos da Lógica Dominada pelo

Serviço. . [S.l.]: Belo Horizonte: UFMG. , mimeo 2014

VERGARA, S. C. Métodos de pesquisa em Administração. São Paulo: Atlas, 2005.

YI, Y.; GONG, T. Customer value co-creation behavior: Scale development and validation. Journal of Business Research, v. 66, p. 1279-1284, 2013. 